\title{
FIVE NEW TRANSITS OF THE SUPER-NEPTUNE HD 149026b
}

\author{
Joshua N. Winn, ${ }^{1}$ Gregory W. Henry, ${ }^{2}$ Guillermo Torres, ${ }^{3}$ and Matthew J. Holman ${ }^{3}$ \\ Received 2007 September 12; accepted 2007 November 12
}

\begin{abstract}
We present new photometry of HD 149026 spanning five transits of its "super-Neptune" planet. In combination with previous data, we improve on the determination of the planet-to-star radius ratio: $R_{p} / R_{\star}=0.0491_{-0.0005}^{+0.0018}$. We find the planetary radius to be $0.71 \pm 0.05 R_{\text {Jup }}$, in accordance with previous theoretical models invoking a high metal abundance for the planet. The limiting error is the uncertainty in the stellar radius. Although we find agreement among four different ways of estimating the stellar radius, the uncertainty remains at $7 \%$. We also present a refined transit ephemeris and a constraint on the orbital eccentricity and argument of pericenter, $e \cos \omega=-0.0014 \pm 0.0012$, based on the measured interval between primary and secondary transits.
\end{abstract}

Subject headings: planetary systems — stars: individual (HD 149026)

Online material: machine-readable table

\section{INTRODUCTION}

Many clues about the processes of planet formation and evolution have been discovered by studying the ensemble properties of exoplanets, such as the "brown dwarf desert" (Halbwachs et al. 2000; Marcy \& Butler 2000) and the tendency for metal-rich stars to have more detectable planets (Santos et al. 2003; Fischer $\&$ Valenti 2005). However, there are also individual exoplanets whose properties bear directly on theories of planet formation and evolution. One of the best examples is the transiting planet HD 149026b (Sato et al. 2005).

Compared to Saturn, HD 149026b has a similar mass but its radius is $15 \%$ smaller, despite the intense irradiation from its parent star that should enlarge the radius. Sato et al. (2005) modeled HD $149026 \mathrm{~b}$ as a dense heavy-element core surrounded by a fluid envelope of solar composition. They found a core mass of $70-80 M_{\oplus}$, which is $65 \%-75 \%$ of the total mass of the planet. This is larger than the canonical core mass of $10-20 M_{\oplus}$ that is expected from the core accretion theory of planet formation (Mizuno 1980; Pollack et al. 1996). The finding of a highly metal-enriched composition was confirmed in models by Fortney et al. (2006), Ikoma et al. (2006), Broeg \& Wuchterl (2007), and Burrows et al. (2007). The latter authors dubbed HD 149026b a "super-Neptune" because the inferred mass fraction of heavy elements is similar to that of an ice giant rather than a gas giant.

Interestingly, the parent star has a rather high metallicity $([\mathrm{Fe} / \mathrm{H}]=+0.36$; Sato et al. 2005). The observation of a large core in such a metal-rich system would seem to support the core accretion theory as opposed to coreless alternatives such as gravitational instability (Boss 1997). However, the larger than expected core mass raises some questions. Why did the growing protoplanet not accrete gas efficiently? Or if it did, what happened to its envelope of light elements? Many scenarios have been proposed: a collision of two massive protoplanets (Sato et al. 2005; Ikoma et al. 2006), in situ formation in a low-pressure nebula (Broeg \& Wuchterl 2007), a viscous and evaporating gas disk

\footnotetext{
1 Department of Physics, and Kavli Institute for Astrophysics and Space Research, Massachusetts Institute of Technology, Cambridge, MA 02139.

2 Center of Excellence in Information Systems, Tennessee State University, 3500 John A. Merritt Boulevard, Box 9501, Nashville, TN 37209.

3 Harvard-Smithsonian Center for Astrophysics, 60 Garden Street, Cambridge, MA 02138.
}

(Ikoma et al. 2006), and a separation of gas from planetesimals at the magnetospheric "X point" (Sato et al. 2005).

More recently, Harrington et al. (2007) found that the $8 \mu \mathrm{m}$ brightness temperature of HD 149026b exceeds its expected blackbody temperature, even if the planet is assumed to absorb all of the incident stellar radiation. In this sense the planet is anomalously hot. The high temperature may result from novel atmospheric or structural properties. Most recently, Torres et al. (2007) announced the discovery of a transiting planet, HAT-P-3b, whose measured mass and radius indicate that it too is highly enriched in heavy elements.

In short, HD $149026 \mathrm{~b}$ seems to be the harbinger of an entirely new kind of planet that current models of planet formation, evolution, and structure cannot accommodate without interesting and possibly exotic modifications. Because of this situation, it is desirable to improve the reliability and the precision of estimates of the system parameters, and especially a key parameter that makes this planet unusual: its small radius.

One can measure the planetary radius by gathering photometry during transits, modeling the light curve, and supplementing the model with external information about the stellar radius. Previously, Sato et al. (2005) analyzed three light curves, and Charbonneau et al. (2006) added three light curves. In this paper we present another five light curves of comparable or higher quality to the previously published data, and we simultaneously model all of the data to derive the most precise planetary, stellar, and orbital parameters that are currently available. We present our observations and data reduction procedure in $\S 2$ and the light-curve modeling procedure in $\S 3$. We provide the results in $\S 4$, along with an extended discussion about the limiting error: the uncertainty in the stellar radius. The final section summarizes the results and speculates on future prospects for improvement.

\section{OBSERVATIONS AND DATA REDUCTION}

We used three of the $0.8 \mathrm{~m}$ automated photometric telescopes (APTs) at Fairborn Observatory to measure the transits of HD 149026b that occurred on UT 2006 April 26, 2006 May 20, 2007 May 3, 2007 June 18, and 2007 June 21. We observed the first three transits with the T11 APT and observed the last two transits simultaneously with the T8, T10, and T11 APTs. All three telescopes are equipped with two temperature-stabilized EMI 9124QB 

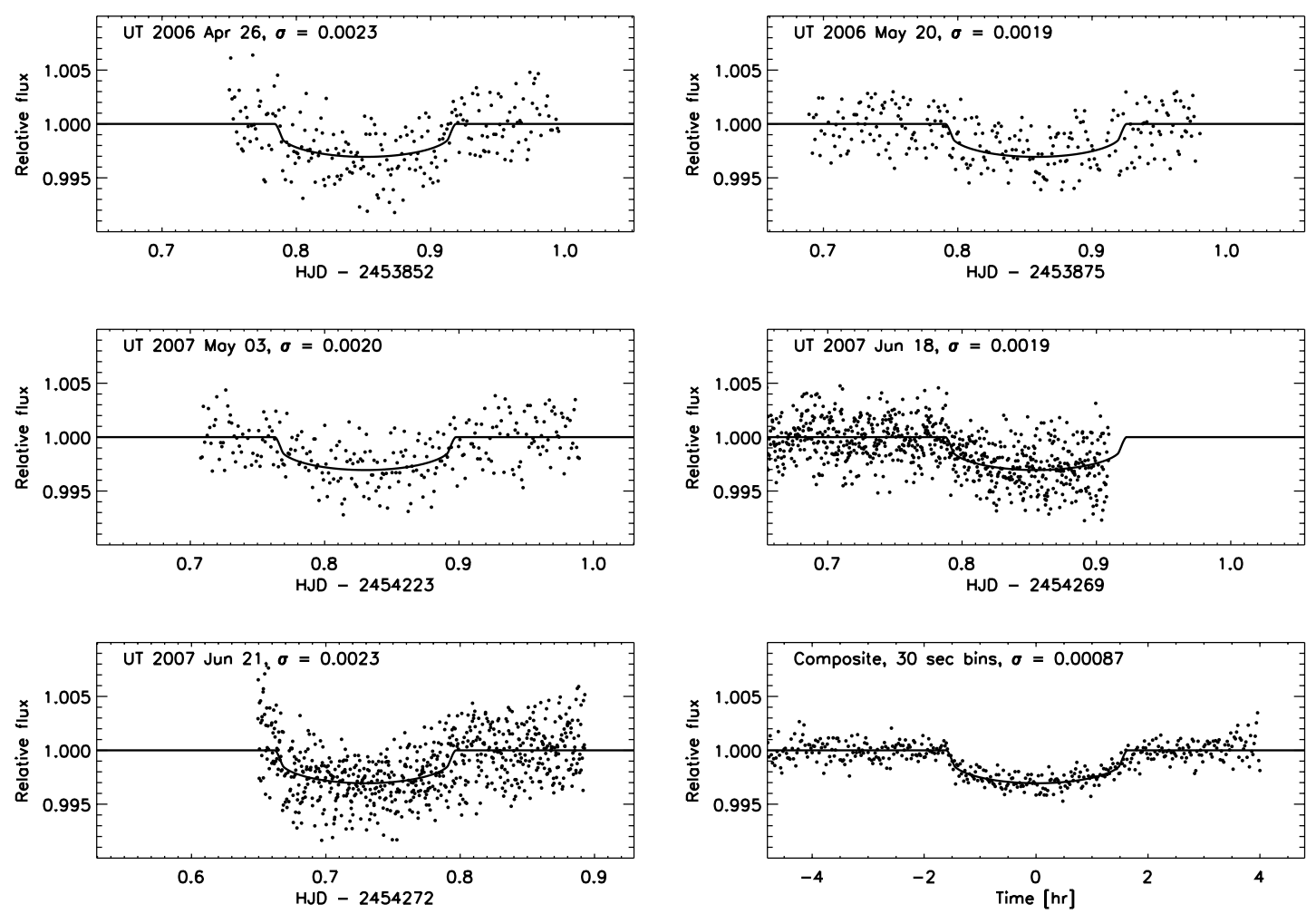

FIG. 1. - Strömgren $(b+y) / 2$ photometry of five new transits of HD 149026 acquired with three of the $0.8 \mathrm{~m}$ APTs at Fairborn Observatory. The bottom right panel is a composite light curve created from the five light curves reported here, as well as the three $(b+y) / 2$ light curves previously published by Sato et al. (2005).

photomultiplier tubes for measuring photon count rates simultaneously through Strömgren $b$ and $y$ filters.

On a given night, each telescope automatically acquired brightness measurements of HD $149026(V=8.15, B-V=0.61)$ and the comparison star HD $149504(V=6.59, B-V=0.44)$, which was previously demonstrated to be stable in brightness at the $0.002 \mathrm{mag}$ level or better (Sato et al. 2005). We also measured the dark count rate and the sky brightness in the vicinity of each star. We used a diaphragm of diameter $45^{\prime \prime}$ for all the integrations. The integration time was $20 \mathrm{~s}$ on the comparison star and $30 \mathrm{~s}$ on the (fainter) target star. We computed the magnitude difference for each pair of target-comparison observations. To increase the signal-to-noise ratio of each measurement, the differential magnitudes from the $b$ and $y$ passbands were averaged, resulting in a differential magnitude for a synthetic $(b+y) / 2$ passband. The typical cadence of the differential magnitude measurements was 1.4 minutes for the first three transits and 1.2 minutes for the last two transits.

For each raw light curve we fitted a linear function of time to the out-of-transit data and divided the data by this function. This was intended to correct for differential air mass effects and other systematic errors in the photometry and also to normalize each light curve to have unit mean flux outside of the transit. (We also tried fitting a function of air mass rather than time, but this gave slightly poorer results.) The final light curves are shown in Figure 1 , and the data are given in Table 1 . The standard deviation of the out-of-transit data is approximately $0.2 \%$ in all cases, which is typical for APT observations of bright stars. For additional information on the telescopes, photometers, observing procedures, data reduction techniques, and typical photometric precision, see Henry (1999) or Eaton et al. (2003). The bottom right panel in Figure 1 is a composite light curve of all eight transits observed by the APTs from Sato et al. (2005) and this paper. The composite light curve was created by subtracting the midtransit time from each of the time stamps and then averaging into $30 \mathrm{~s}$ bins. It is shown here for display purposes only; the fitting procedure described in $\S 3$ was carried out on the unbinned data.

\section{DETERMINATION OF SYSTEM PARAMETERS}

We fitted all of our new photometric data jointly with the three $(b+y) / 2$ light curves presented by Sato et al. (2005) and the $g$ and $r$ light curves presented by Charbonneau et al. (2006). ${ }^{4} \mathrm{We}$ used a parameterized model based on a two-body circular orbit. The orbit is specified by the masses of the star and planet $\left(M_{\star}\right.$ and $M_{p}$ ), the inclination with respect to the sky plane $(i)$, the orbital period $(P)$, and a particular midtransit time $\left(T_{c}\right)$. The star and planet are taken to be spheres with radii $R_{\star}$ and $R_{p}$, respectively, and when their sky-projected centers are within $R_{\star}+R_{p}$ of one another we use the Mandel \& Agol (2002) formulas to compute

\footnotetext{
${ }^{4}$ We did not include the $V$-band light curve of Charbonneau et al. (2006) because of its comparatively large errors and sparse time sampling.
}

TABLE 1

РнотометRY OF HD 149026

\begin{tabular}{|c|c|c|}
\hline Heliocentric Julian Date & Relative Flux & Uncertainty \\
\hline $2,453,852.75015 \ldots \ldots \ldots \ldots \ldots \ldots \ldots$ & 1.0031 & 0.0027 \\
\hline $2,453,852.75115 \ldots \ldots \ldots \ldots \ldots \ldots \ldots \ldots \ldots \ldots \ldots$ & 1.0061 & 0.0027 \\
\hline $2,453,852.75215 \ldots \ldots \ldots \ldots \ldots \ldots \ldots \ldots \ldots \ldots \ldots$ & 1.0023 & 0.0027 \\
\hline
\end{tabular}

Notes.-Table 1 is published in its entirety in the electronic edition of the Astrophysical Journal. A portion is shown here for guidance regarding its form and content. The data were obtained with the automatic photometric telescopes (APTs) at Fairborn Observatory. Differential magnitudes were measured in the Strömgren $b$ and $y$ passbands, and the $b$ and $y$ results were averaged. The time stamps represent the heliocentric Julian date at the time of midexposure. The uncertainties include the "red noise" correction described in $\S 3$. 
TABLE 2

HD 149026: Transit Light Curve Parameters

\begin{tabular}{|c|c|c|c|}
\hline Parameter & Value & 68\% Upper Limit & $68 \%$ Lower Limit \\
\hline$R_{p} / R_{\star}$. & 0.0491 & +0.0018 & -0.0005 \\
\hline$a / R_{\star} \ldots \ldots \ldots$ & 7.11 & +0.03 & -0.81 \\
\hline$i(\operatorname{deg}) \ldots \ldots \ldots \ldots$ & 90.0 & +3.1 & -3.1 \\
\hline$b \equiv a \cos i / R_{\star} \ldots \ldots \ldots \ldots \ldots \ldots$ & 0.00 & +0.36 & -0.36 \\
\hline Total transit duration $(\mathrm{hr})^{\mathrm{a}} \ldots \ldots \ldots \ldots$ & 3.254 & +0.057 & -0.028 \\
\hline Ingress or egress duration $(\mathrm{hr})^{\mathrm{b}}$. & 0.153 & +0.052 & -0.002 \\
\hline
\end{tabular}

Notes.-Results of fitting 10 light curves: three light curves $[(b+y) / 2]$ from Sato et al. (2005), two light curves ( $g$ and $r$ ) from Charbonneau et al. (2006), and five light curves $[(b+y) / 2]$ from this work. Not all of the parameters are independent. One may regard $R_{p} / R_{\star}, a / R_{\star}$, and $i$ as the basic parameters from which the other results in this table may be derived.

${ }^{\text {a }}$ Defined as the time between first and fourth contacts (i.e., between the moments when the projected planetary and stellar disks are externally tangent).

${ }^{b}$ Defined as the time between first and second contacts (i.e., the duration over which the projected planetary disk crosses the stellar limb, from external tangency to internal tangency). In our model, the ingress and egress durations are equal.

the flux decrement due to the partial blockage of the limbdarkened stellar surface. This is the same code that has been developed for the Transit Light Curve project (Holman et al. 2006; Winn et al. 2007). For HD 149026, we assumed the limbdarkening law to be linear, with a coefficient given by Claret (2000) for a star of the appropriate temperature and surface gravity. For the $(b+y) / 2$ data we used a coefficient of 0.712 , which is the mean of the tabulated $b$ - and $y$-coefficients.

Not all of the parameters listed above can be determined from transit photometry alone. One set of parameters that can be determined from an individual light curve is $T_{c}, R_{p} / R_{\star}, a / R_{\star}$, and $i$, where $a$ is the semimajor axis. Our approach was to fix $M_{\star}, M_{p}$, and $P$ at previously determined values (thereby fixing $a$ through Kepler's third law), and then fit for $R_{p}, R_{\star}, i$, and $T_{c}$. The results for $R_{\star}$ and $R_{p}$ are specific to the choice of $M_{\star}$, but they scale as $M_{\star}^{1 / 3}$ because $a \propto M_{\star}^{1 / 3}$ when the uncertainty in $P$ is negligible, as it is here. We assumed $M_{\star}=1.3 M_{\odot}$, following Sato et al. (2005), a choice that was subsequently corroborated by our analysis of the observable stellar properties and the results for $a / R_{\star}$ (see $\left.\S 4.2\right)$.

The fitting statistic was

$$
\chi^{2}=\sum_{j=1}^{N_{f}}\left[\frac{f_{j}(\mathrm{obs})-f_{j}(\mathrm{calc})}{\sigma_{j}}\right]^{2},
$$

where $N_{f}$ is the number of flux measurements, $f_{j}(\mathrm{obs})$ is the flux observed at time $j, \sigma_{j}$ controls the weights of the data points, and $f_{j}$ (calc) is the calculated flux. Experience has shown that the data weights $\sigma_{j}$ should account not only for the single-measurement precision but also the time-correlated ("red") noise that afflicts most time-series photometry (see, e.g., Gillon et al. 2006). The most important timescale in a transit light curve is the $\sim 10$ minute duration of the ingress and egress, since the resolution of ingress and egress is what permits the determination of $a / R_{\star}$ and $i$ in addition to $R_{p} / R_{\star}$. To assess the noise on this timescale, we first calculated the standard deviation of the unbinned out-of-transit data $\left(\sigma_{1}\right)$ for each light curve. Then we averaged the out-of-transit data into 10 minute bins consisting of $N$ data points, where $N$ depended on the observing cadence, and recalculated the standard deviation $\left(\sigma_{\mathrm{N}}\right)$. In the absence of red noise, one would expect $\sigma_{N}=\sigma_{1} / \sqrt{N}$, but in practice $\sigma_{N}$ was larger than $\sigma_{1} / \sqrt{N}$ by some factor $\beta$. Therefore, we set the data weights equal to $\beta \sigma_{1}$. The results for $\beta$ ranged from 1.05 to 1.27 .
We used a Markov chain Monte Carlo (MCMC) algorithm to determine the best-fitting parameter values and confidence intervals. This algorithm delivers an estimate of the a posteriori joint probability distribution for all of the parameters (see Holman et al. 2006 or Winn et al. 2007 for more details). For each parameter we took the mode of the distribution after marginalizing over all other parameters to be the "best value." We defined the $68 \%$ confidence limits $p_{\mathrm{lo}}$ and $p_{\mathrm{hi}}$ as the values between which the integrated probability is $68 \%$, and for which the two integrals from $p_{\min } \rightarrow p_{\text {lo }}$ and $p_{\text {hi }} \rightarrow p_{\max }$ were equal.

At first, the preceding computations were performed using free parameters for both the orbital period and a single midtransit time. Thus, the transits were required to be spaced by integral multiples of a fixed period. However, we also wanted to measure the individual transit times in order to search for variations that might be indicative of additional bodies in the planetary system (Agol et al. 2005; Holman \& Murray 2005). To do this we fixed $R_{p}, R_{\star}$, and $i$ at the best values determined in the first step, and then performed a three-parameter fit of each individual light curve. The parameters were $T_{c}$ along with the zero point and slope of the linear function that was used to correct the out-of-transit data. (Fixing the values of $R_{p}, R_{\star}$, and $i$ is justified because the errors in those parameters are not correlated with the error in the transit time.) We did this not only for the five new light curves, but also for the five previously published light curves, to provide consistency in the treatment of errors. We then used these transit-time measurements to refine the estimates of $P$ and $T_{c}$ (see $\S 4.3$ ). For our final results for the photometric parameters $R_{p} / R_{\star}, a / R_{\star}$, and $i$, we reran the MCMC algorithm on the entire data set, using fixed values of $P$ and $T_{c}$ from our refined ephemeris.

\section{RESULTS}

The results of the light-curve analysis are given in Table 2 and discussed in $\S 4.1$. The transit times of the individual light curves are given in Table 3, and in $\S 4.2$ we use those times to derive a new transit ephemeris. We also use the new ephemeris along with a previously measured midpoint of a secondary eclipse to place an upper bound on one aspect of the orbital eccentricity. In order to derive the actual planetary radius (as opposed to the planet-to-star radius ratio) one must supplement the light-curve analysis with external information about the stellar radius or mass. In $\S 4.3$ we investigate four different methods to estimate the stellar radius, the results of which are given in Table 4 . The planetary parameters 
TABLE 3

HD 149026: Midtransit Times

\begin{tabular}{|c|c|c|c|}
\hline Telescope & $\begin{array}{l}\text { Epoch } \\
\quad E\end{array}$ & $\begin{array}{l}\text { Mid-Transit Time } \\
\text { (HJD) }\end{array}$ & $\begin{array}{c}\text { Uncertainty } \\
\text { (days) }\end{array}$ \\
\hline T11 $0.8 \mathrm{~m} \mathrm{APT...}$ & -267 & $2,453,504.8707$ & 0.0022 \\
\hline 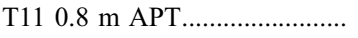 & -259 & $2,453,527.8732$ & 0.0021 \\
\hline FLWO $1.2 \mathrm{~m}$ & -259 & $2,453,527.8722$ & 0.0022 \\
\hline T8, T10, T11 $0.8 \mathrm{~m}$ APTs....... & -258 & $2,453,530.7517$ & 0.0031 \\
\hline 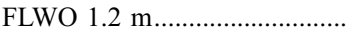 & -250 & $2,453,553.7583$ & 0.0013 \\
\hline 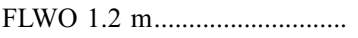 & -250 & $2,453,553.7598$ & 0.0045 \\
\hline 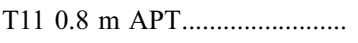 & -146 & $2,453,852.8514$ & 0.0024 \\
\hline 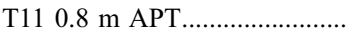 & -138 & $2,453,875.8600$ & 0.0050 \\
\hline 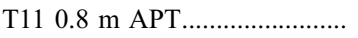 & -17 & $2,454,223.8355$ & 0.0070 \\
\hline T8, T10, T11 0.8 m APTs....... & -1 & $2,454,269.8554$ & 0.0021 \\
\hline T8, T10, T11 $0.8 \mathrm{~m}$ APTs....... & 0 & $2,454,272.7293$ & 0.0017 \\
\hline
\end{tabular}

Notes.-Based on these measurements we derived a transit ephemeris $T_{c}(E)=$ $T_{c}(0)+E P$ with $T_{c}(0)=2,454,272.7301(13)$ (HJD) and $P=2.8758882(61)$ days, where the numbers in parentheses indicate the $1 \sigma$ uncertainty in the final two digits.

derived from the light curves and the stellar radius estimates are given in Table 5.

\subsection{Photometric Parameters}

The a posteriori probability distributions for $R_{p} / R_{\star}, a / R_{\star}$, and $i$ are shown in Figure 2. The most well constrained of these three basic light-curve parameters is the radius ratio, $R_{p} / R_{\star}=$ $0.0491_{-0.0005}^{+0.0018}$, with a precision of approximately $2.3 \%$. The radius ratio is determined largely by the observed transit depth, which is the smallest among all of the 20 transiting planets known to date. While there are smaller planets, such as the Neptune-sized GJ 436b (Gillon et al. 2007), they all orbit smaller stars, making their radius ratios and transit depths larger than that of HD 149026.

Less well constrained are $a / R_{\star}$ and $i$, the parameters that depend on the observed durations of the ingress, egress, and total phases of the transit. Table 2 gives the results for those parameters, as well as the impact parameter and the durations, which can be derived in terms of $R_{p} / R_{\star}, a / R_{\star}$, and $i$. The data are consistent with impact parameters ranging from -0.36 to 0.36 . As with any eclipsing binary system, the data cannot distinguish between positive and negative impact parameters; the probability distributions are perfectly symmetric about $b=0$ and $i=90^{\circ}$. In this case the peak probability occurs at the central values $b=0, i=90^{\circ}$. The quantity $a / R_{\star}$ has a highly asymmetric error bar. The observed duration of the entire transit event enforces the upper limit on $a / R_{\star}$, while the ratio of the ingress (or egress) duration to the total duration enforces the lower limit on $a / R_{\star}$.

Our findings are consistent with the two previous light-curve analyses, by Sato et al. (2005) and Charbonneau et al. (2006), although our analysis method is different in several ways besides the use of an expanded data set. First, we have attempted to account for time-correlated noise in the photometry, which was neglected in the previous analyses. Second, unlike the previous authors, we have not incorporated any a priori constraints on the stellar properties into our fitting statistic. We made this choice in order to clarify what information is derived from the light curves themselves; for example, the previous works did not call attention to the results for $R_{p} / R_{\star}$ even though that parameter is more precisely known than either $R_{p}$ or $R_{\star}$. In addition, our analysis method provides an estimate of $a / R_{\star}$ that is independent of any assumptions about the parent star, except for the very weak dependence on the chosen limb-darkening parameter. This is useful because $a / R_{\star}$ can be used
TABLE 4

HD 149026: Stellar Radius

\begin{tabular}{ccc}
\hline \hline $\begin{array}{c}\text { Radius } \\
\left(R_{\odot}\right)\end{array}$ & Method & Reference \\
\hline $1.46 \pm 0.10 \ldots \ldots \ldots \ldots .$. & Stefan-Boltzmann law & 1,2 \\
$1.515 \pm 0.096 \ldots \ldots \ldots \ldots$ & Spectral energy distribution fit & 3 \\
$1.368_{-0.083}^{+0.124 \ldots \ldots \ldots \ldots \ldots . .}$ & Yonsei-Yale isochrone fit & 2 \\
$1.35_{-0.02}^{+0.17} \ldots \ldots \ldots \ldots \ldots \ldots$ & Kepler's law with stellar mass prior ${ }^{\mathrm{a}}$ & 2 \\
\hline
\end{tabular}

${ }^{\mathrm{a}}$ Using $M_{\star}=1.30 \pm 0.06$, based on the Yonsei-Yale isochrone fit to $a / R_{\star}$ and $T_{\text {eff }}$.

ReFERENCES.-(1) Sato et al. 2005; (2) This work; (3) Masana et al. 2006.

to determine the stellar mean density (Seager \& Mallen-Ornelas 2003; Sozzetti et al. 2007: Holman et al. 2007):

$$
\rho_{\star}=\frac{3 \pi}{G P^{2}}\left(\frac{a}{R_{\star}}\right)^{3}-\rho_{p}\left(\frac{R_{p}}{R_{\star}}\right)^{3} .
$$

The last term in this expression may be neglected in this case because $\rho_{p} \sim \rho_{\star}$ and $\left(R_{p} / R_{\star}\right)^{3} \sim 10^{-4}$. Our independent estimate of $\rho_{\star}$ is useful in characterizing the parent star, as described below.

\subsection{The Stellar Radius}

To determine the quantity of intrinsic interest, $R_{p}$, we can multiply our result for $R_{p} / R_{\star}$ by a value of $R_{\star}$ obtained by other means. We have investigated four different methods for determining $R_{\star}$ :

Stefan-Boltzmann law.-The bolometric luminosity, effective temperature, and photospheric radius of HD 149026 are related via $L_{\mathrm{bol}}=4 \pi R_{\star}^{2} \sigma T_{\text {eff }}^{4}$. We use the Hipparcos parallax and apparent magnitude $(\pi=12.68 \pm 0.79$ mas, $V=8.15 \pm 0.02$; Perryman et al. 1997) to compute the absolute $V$ magnitude, apply a bolometric correction of $-0.027 \pm 0.014$ (Flower 1996), and use the spectroscopically determined $T_{\text {eff }}=6147 \pm 50 \mathrm{~K}$ (Sato et al. 2005). The result is $R_{\star}=1.46 \pm 0.10 R_{\odot}$. This is essentially identical to the value quoted by Sato et al. (2005), who used the same method.

Spectral energy distribution fit.-Masana et al. (2006) presented an alternative means of estimating the effective temperature and bolometric correction, using VJHK photometry. They also provided radius estimates for many nearby stars based on this technique. Using the Hipparcos parallax and $V$ magnitude along with 2MASS near-infrared photometry, their result for HD 149026 is $R_{\star}=1.515 \pm 0.096 R_{\odot}$.

Yonsei-Yale isochrone fit. - Stellar evolutionary models may be used to estimate the mass, radius, and age of a star with a given effective temperature, luminosity (or gravity), and metallicity. We used the Yonsei-Yale models (Yi et al. 2001; Demarque et al. 2004) because they are conveniently provided with tools for interpolating isochrones in both age and metallicity. For the effective temperature, we used $T_{\text {eff }}=6160 \pm 50 \mathrm{~K}$, a weighted mean of the results of Sato et al. (2005) and Masana et al. (2006). We used the photometric result for $a / R_{\star}$ as our proxy for surface gravity, and we explored the range ${ }^{5}$ of metallicities $[\mathrm{Fe} / \mathrm{H}]=0.36 \pm 0.08$. For each metallicity we considered a range of ages from 0.1 to $14 \mathrm{Gyr}$, in steps of $0.1 \mathrm{Gyr}$. We interpolated the isochrones using a fine mass grid and compared the points with the measured values of $T_{\text {eff }}$ and $a / R_{\star}$. We computed $\chi^{2}$ at each point based on the

\footnotetext{
${ }^{5}$ Sato et al. (2005) reported a metallicity of $[\mathrm{Fe} / \mathrm{H}]=+0.36$ with an internal uncertainty of 0.05 . To be conservative, we adopted a somewhat larger uncertainty of 0.08 , recognizing that different methods for determining the metallicity often produce systematic differences of this size.
} 
TABLE 5

HD 149026: Planetary Parameters

\begin{tabular}{|c|c|c|}
\hline Parameter & Value & Method \\
\hline$M_{p}\left(M_{\text {Jup }}\right)$ & $0.36 \pm 0.03$ & Spectroscopic orbit ${ }^{\mathrm{a}}$ \\
\hline 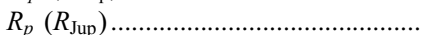 & $0.71 \pm 0.05$ & $R_{p} / R_{\star}$ from light curves and $R_{\star}=1.45 \pm 0.10$ \\
\hline $\log g_{p}(\mathrm{cgs}) \ldots \ldots \ldots \ldots \ldots \ldots \ldots \ldots \ldots \ldots \ldots$ & $3.357_{-0.130}^{+0.008}$ & Light curve and spectroscopic orbit ${ }^{\mathrm{b}}$ \\
\hline 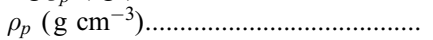 & $1.25 \pm 0.28$ & $M_{p}, R_{p}$ given above \\
\hline Semimajor axis, $a(\mathrm{AU}) \ldots \ldots \ldots \ldots \ldots \ldots \ldots$ & $0.0432 \pm 0.0006$ & Kepler's law ${ }^{c}$ \\
\hline$e \cos \omega \ldots \ldots \ldots \ldots \ldots \ldots$ & $-0.0014 \pm 0.0012$ & Timing of secondary eclipse ${ }^{\mathrm{d}}$ \\
\hline
\end{tabular}

${ }^{\text {a }}$ Using $K=43.3 \pm 1.2 \mathrm{~m} \mathrm{~s}^{-1}$ from Sato et al. (2005), $P$ from Table 2, and $M_{\star}=1.30 \pm 0.06$, based on the Yonsei-Yale isochrone fit to $a / R_{\star}$ and $T_{\text {eff }}$.

${ }^{\mathrm{b}}$ Using $K=43.3 \pm 1.2 \mathrm{~m} \mathrm{~s}^{-1}$ from Sato et al. (2005), $P$ from Table 2, and $i, a / R_{p}$ from the light-curve analysis. This method is described in detail by Southworth et al. (2007) and Sozzetti et al. (2007).

${ }^{c}$ Using $P$ from Table 2 and $M_{\star}=1.30 \pm 0.06$, based on the Yonsei-Yale isochrone fit to $a / R_{\star}$ and $T_{\text {eff }}$.

${ }^{\mathrm{d}}$ Using the secondary eclipse time HJD $=2,453,606.960 \pm 0.001$ from Harrington et al. (2007) and the ephemeris given in Table 2 (after correcting for the $43 \mathrm{~s}$ light travel time across the orbit).

modeled and observed values of $T_{\text {eff }}, a / R_{\star}$, and metallicity. Then we weighted the points by $\exp \left(-\chi^{2} / 2\right)$ and applied an additional weighting to take into account the density of stars on each isochrone, assuming a Salpeter initial mass function. The "bestfitting" stellar properties were taken to be the weighted mean of the properties of all the points. For more details and other applications of this analysis, see, Torres et al. (2008). For HD 149026, the results are $M_{\star}=1.294_{-0.050}^{+0.060} M_{\odot}, L_{\star}=2.430_{-0.348}^{+0.533} L_{\odot}$, and $R_{\star}=1.368_{-0.083}^{+0.124} R_{\odot}$. Similar results were obtained when the spectroscopically determined value of $\log g$ was used instead of $a / R_{\star}$. The theoretical isochrones and the observational constraints are shown in Figure 3.

Kepler's law with stellar mass prior.-As mentioned earlier, the quantity $a / R_{\star}$ that is determined from the transit photometry can be used to find $\rho_{\star}$ (eq. [2]). With an a priori estimate of $M_{\star}$, one may use $\rho_{\star}$ to determine $R_{\star}$. Taking $M_{\star}=1.30 \pm 0.06 M_{\odot}$ based on the isochrone fit described above, we find $R_{\star}=$ $1.35_{-0.02}^{+0.17} R_{\odot}$.
All of the results for the stellar radius are summarized in Table 4. They are all consistent with one another at the $1 \sigma$ level, with a weighted mean of $1.45 R_{\odot}$. However, it must be emphasized that while the methods are different, they are not wholly independent. The first two methods both rely on the Hipparcos parallax, which is the largest source of error in both cases. The latter two methods both rely on the Yonsei-Yale stellar evolutionary models. For this reason we cannot say confidently that the uncertainty in $R_{\star}$ is any smaller than the uncertainty in each of the individual measurements, although the mutual agreement is certainly reassuring. In what follows we adopt the consensus value $R_{\star}=1.45 \pm 0.10 R_{\odot}$, the same value used in the previous light-curve analyses.

Assuming a Gaussian error distribution for $R_{\star}$, and the error distribution for $R_{p} / R_{\star}$ obtained from our light-curve analysis, we find the planetary radius to be $R_{p}=0.71 \pm 0.05 R_{\text {Jup }}$. This can be compared to the previously published results of $0.725 \pm$ $0.050 R_{\text {Jup }}$ (Sato et al. 2005) and $0.726 \pm 0.064 R_{\text {Jup }}$ (Charbonneau et al. 2006), keeping in mind our different method of analysis and
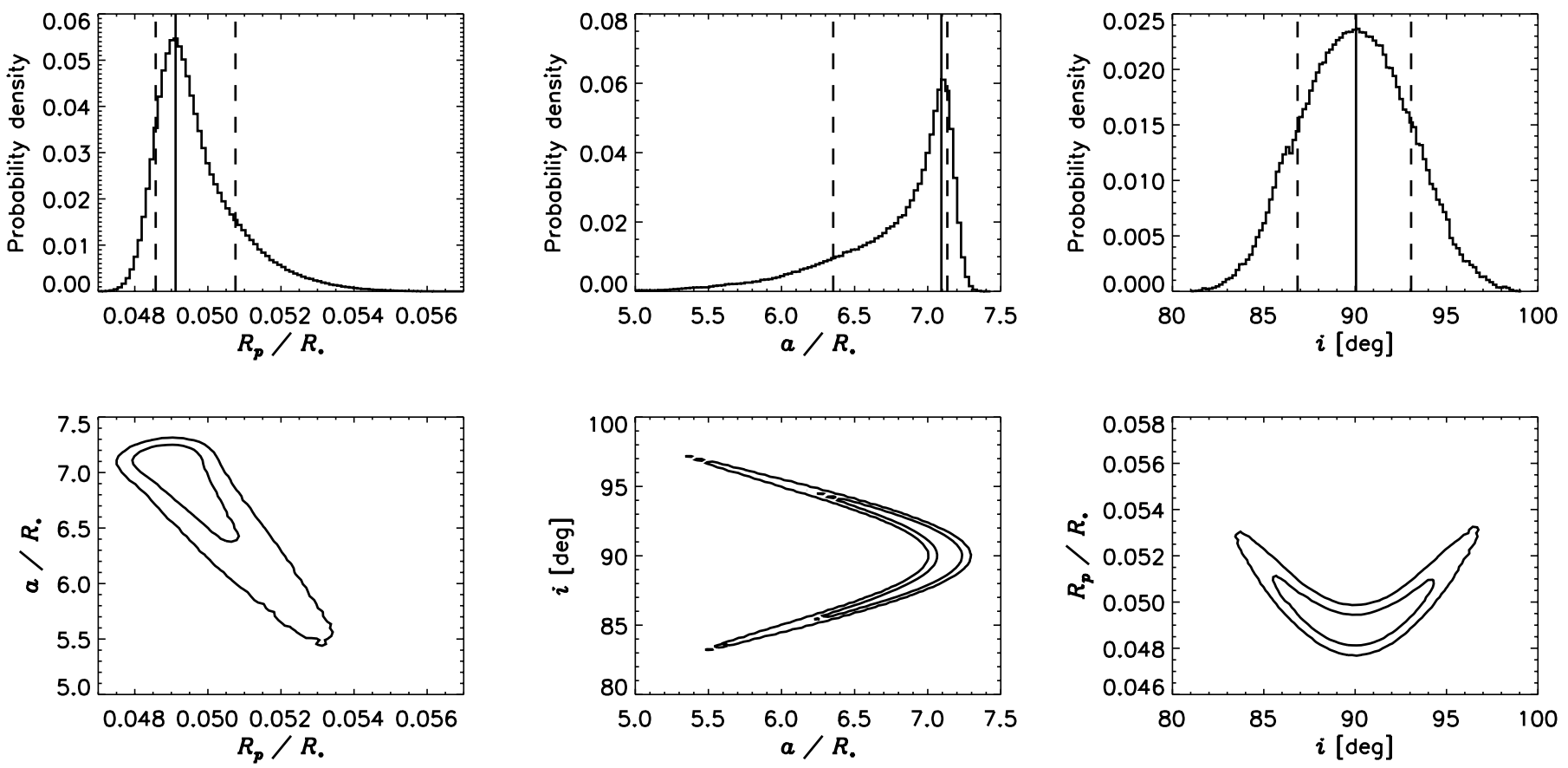

FIG. 2.-Estimated a posteriori probability distributions from the joint fit to the transit light curves. The top panels show the single-variable distributions, in which the mode is marked with a solid line and the $68 \%$ confidence limits with dashed lines. The bottom panels show the two-dimensional distributions, in which the contours mark the $68 \%$ and $95 \%$ confidence limits. 


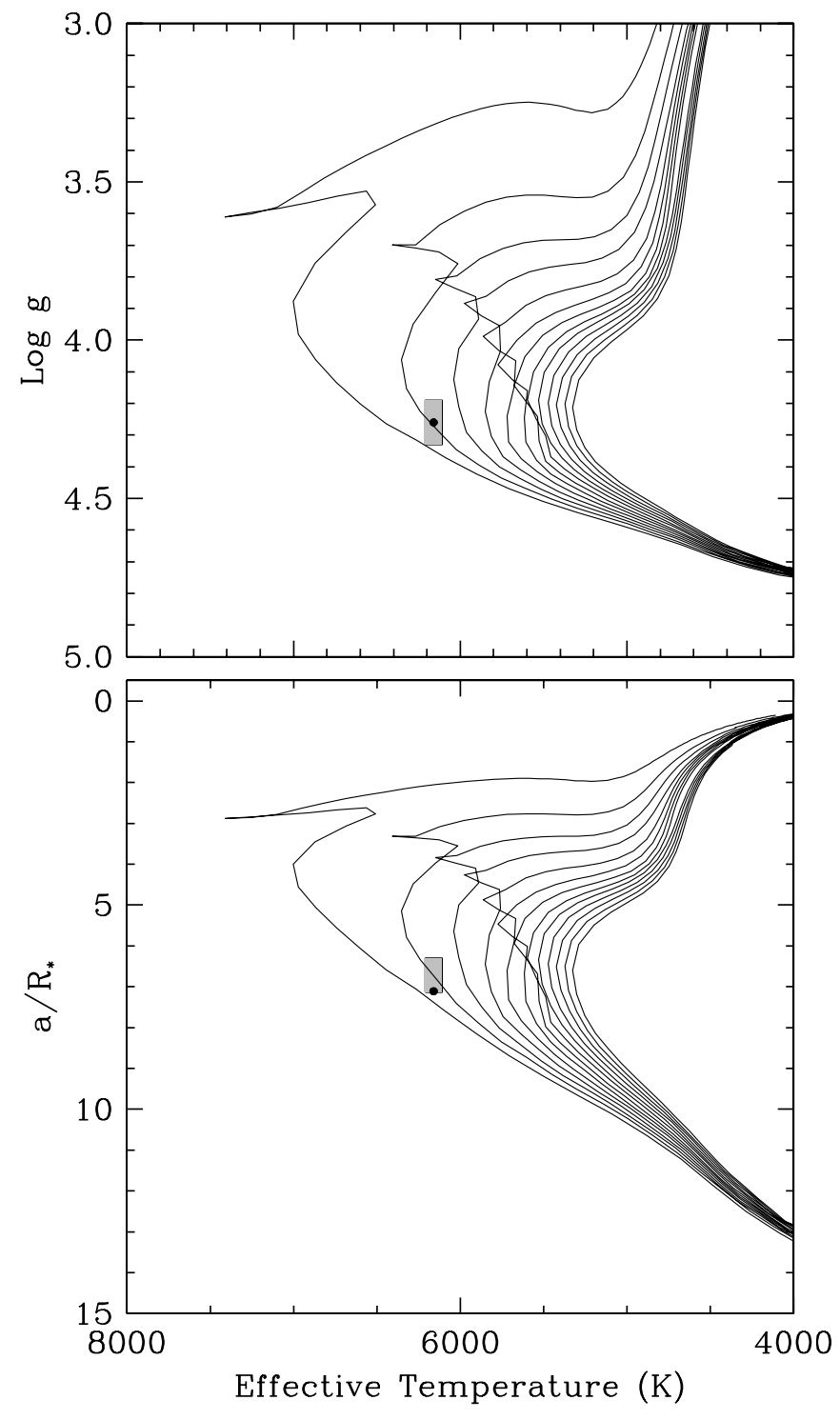

FIG. 3.-Model isochrones from the Yonsei-Yale series by Yi et al. (2001) and Demarque et al. (2004), corresponding to ages of 1-14 Gyr (left to right) for the measured composition of $[\mathrm{Fe} / \mathrm{H}]=+0.36$, along with the observational constraints. Top: The vertical axis is $\log g$, and the shaded box shows the $1 \sigma$ range based on the spectroscopically determined value of $\log g$. Bottom: The vertical axis is $a / R_{\star}$, which is proportional to the cube root of the stellar mean density (see eq. [2]). The shaded box shows the $1 \sigma$ range based on the photometrically determined value of $a / R_{\star}$.

treatment of observational errors. The results are all in agreement. Indeed, the differences are smaller than one would expect from Gaussian statistics, given the quoted error bars, although we note that 5 of the 10 light curves that we fitted were taken from those previous works. The precision in $R_{p}$ is not improved because the limiting error is the uncertainty in $R_{\star}$, which is unchanged.

\subsection{Transit Times}

For planning future observations of this system it is important to be able to predict transit times as precisely as possible. We used all of the transit times given in Table 3 to calculate a photometric ephemeris for this system,

$$
T_{c}(E)=T_{c}(0)+E P
$$

where $T_{c}$ is the transit midpoint, $E$ is the integral transit epoch, and $P$ is the orbital period. The linear fit had $\chi^{2} / N_{\mathrm{dof}}=0.63$ and

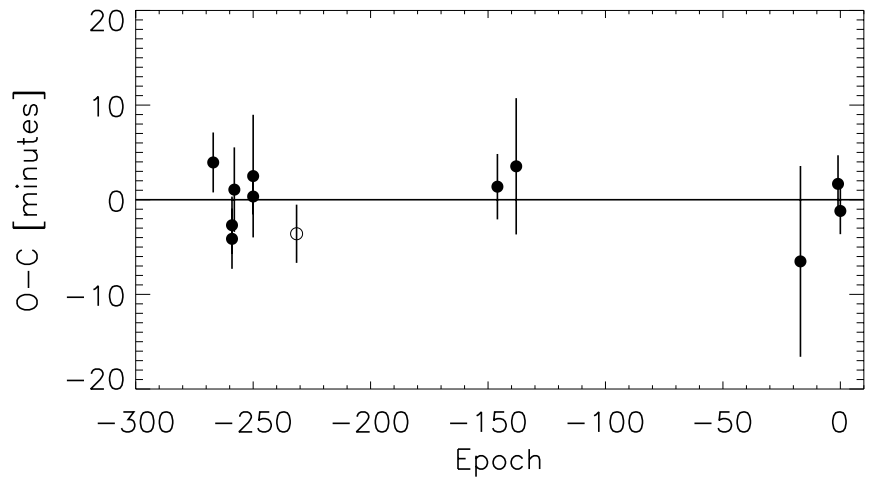

FIG. 4.-Transit timing residuals for HD 149026b. The calculated times, using the ephemeris derived in $\S 4.3$, have been subtracted from the observed times. The filled symbols represent observations of transits. The open symbol represents the observation of the secondary eclipse by Harrington et al. (2007). The secondary eclipse datum was not used in the fit.

$N_{\text {dof }}=9$, suggesting that the errors quoted in Table 3 have been somewhat overestimated. The results are

$$
\begin{gathered}
T_{c}(0)=2,454,272.7301 \pm 0.0013(\mathrm{HJD}), \\
P=2.8758882 \pm 0.0000061 \text { days. }
\end{gathered}
$$

Our value for the orbital period is in agreement with the previously published values and is about 25 times more precise. Figure 4 is the $O-C$ (observed minus calculated) diagram for the transit times.

For a circular orbit, successive transits and secondary eclipses should be spaced by exactly half an orbital period. Recently, Harrington et al. (2007) observed a secondary eclipse of HD 149026 with the Spitzer Space Telescope, allowing the assumption of a circular orbit to be checked. In the presence of a small but nonzero orbital eccentricity, the time difference between the midpoint of secondary eclipse, $T_{\mathrm{sec}}$, and the time of transit, $T_{\mathrm{tra}}$, is

$$
T_{\text {sec }}-T_{\text {tra }} \approx \frac{P}{2}\left(1+\frac{4}{\pi} e \cos \omega\right),
$$

where $\omega$ is the argument of pericenter (Kallrath \& Milone 1999, p. 62). Harrington et al. (2007) measured the midpoint of a secondary eclipse to be HJD 2,453,606.960 \pm 0.001 , represented by the open circle in Figure 4. The timing offset of equation (6) is $-3.6 \pm 3.1$ minutes, corresponding to $e \cos \omega=-0.0014 \pm$ 0.0012 . The orbit does indeed appear to be nearly circular, as one would expect from the dissipative effects of stellar and planetary tidal interactions.

\section{DISCUSSION AND SUMMARY}

We have presented five new transit light curves of the exoplanet HD $149026 \mathrm{~b}$ and analyzed them along with five previously published light curves. The joint analysis has resulted in much more precise determinations of the orbital period and transit ephemerides and also in a more precise value of the planet-to-star radius ratio. In some cases, this ratio is of primary interest, such as inferring the brightness temperature of the planet from the depth of a secondary eclipse (Harrington et al. 2007), or testing for any wavelength dependence in the radius ratio as a means of identifying planetary atmospheric features (see, e.g., Charbonneau et al. 2002).

However, when it comes to understanding the interior structure of the planet, the quantity of primary interest is $R_{p}$ itself, and here we can offer no significant improvement. The limiting error 
is the $7 \%$ uncertainty in the stellar radius. This error was not reduced by acquiring more light curves, although we did find agreement between the results of four different (and intertwined) methods for estimating the stellar radius using all of the available data. Thus, we leave unchanged the interpretation of this planet as a being unexpectedly small for its mass and likely to be highly enriched in heavy elements (Sato et al. 2005; Fortney et al. 2006; Ikoma et al. 2006; Burrows et al. 2007).

Further improvement will depend on progress in measuring the stellar radius. Baines et al. (2007) recently used optical interferometry to measure the angular diameter of the planet-hosting star HD 189733 and combined it with the Hipparcos parallax to measure the stellar radius. For HD 149026, similar observations are not likely to result in a more precise value of the stellar radius, at least not in the near future. This is not only because of the $6 \%$ uncertainty in the parallax, but also because the expected angular diameter is only $\approx 180 \mu$ as, which is only $7-8$ times larger than the measurement error that was achieved for HD 189733.

Supposing the parallax were known with $10 \mu$ as precision (as one might hope from a space-based interferometric mission), the error in the Stefan-Boltzmann method for determining $R_{\star}$ would be reduced to $2.7 \%$. The limiting errors in that case would arise from the effective temperature and bolometric correction. In the nearer term a possible path forward is the continued acquisition of high-quality transit photometry, in order to improve on our measurement of $a / R_{\star}$ and thereby establish the stellar mean density with greater precision. At fixed mean density, $R_{\star}$ varies as $M_{\star}^{1 / 3}$, and our application of the Yonsei-Yale models to HD 149026 suggests that the stellar mass has already been pinned down to within $4.6 \%$. If $a / R_{\star}$ were known exactly, the fractional error in the stellar radius would be approximately $1.5 \%$ (i.e., one-third as large as the fractional error in the stellar mass). In effect, transit photometry measures $M_{\star} / R_{\star}^{3}$, and the stellar models generally constrain a different combination of $M_{\star}$ and $R_{\star}$ (see, e.g., Cody \& Sasselov 2002). We encourage observers to be persistent in gathering additional seasons of ground-based photometry and look forward to the results of space-based photometry for this system.

We are grateful to the anonymous referee for a thorough and helpful review of the manuscript. G. W. H. acknowledges support from NSF grant HRD-9706268 and NASA grant NNX06AC14G. G. T. acknowledges partial support for this work from NASA grant NNG04LG89G.
Agol, E., Steffen, J., Sari, R., \& Clarkson, W. 2005, MNRAS, 359, 567

Baines, E. K., van Belle, G. T., ten Brummelaar, T. A., McAlister, H. A., Swain, M., Turner, N. H., Sturmann, L., \& Sturmann, J. 2007, ApJ, 661, L195

Boss, A. P. 1997, Science, 276, 1836

Broeg, C., \& Wuchterl, G. 2007, MNRAS, 376, L62

Burrows, A., Hubeny, I., Budaj, J., \& Hubbard, W. B. 2007, ApJ, 661, 502

Charbonneau, D., Brown, T. M., Noyes, R. W., \& Gilliland, R. L. 2002, ApJ, 568,377

Charbonneau, D., et al. 2006, ApJ, 636, 445

Claret, A. 2000, A\&A, 363, 1081

Cody, A. M., \& Sasselov, D. D. 2002, ApJ, 569, 451

Demarque, P., Woo, J.-H., Kim, Y.-C., \& Yi, S. K. 2004, ApJS, 155, 667

Eaton, J. A., Henry, G. W., \& Fekel, F. C. 2003, in The Future of Small Telescopes in the New Millennium, Vol. II-The Telescopes We Use, ed. T. D. Oswalt (Dordrecht: Kluwer), 189

Fischer, D. A., \& Valenti, J. 2005, ApJ, 622, 1102

Flower, P. J. 1996, ApJ, 469, 355

Fortney, J. J., Saumon, D., Marley, M. S., Lodders, K., \& Freedman, R. S. 2006, ApJ, 642, 495

Gillon, M., Pont, F., Moutou, C., Bouchy, F., Courbin, F., Sohy, S., \& Magain, P. 2006, A\&A, 459, 249

Gillon, M., et al. 2007, A\&A, 472, L13

Halbwachs, J. L., Arenou, F., Mayor, M., Udry, S., \& Queloz, D. 2000, A\&A, 355,581

Harrington, J., Luszcz, S., Seager, S., Deming, D., \& Richardson, L. J. 2007, Nature, 447, 691

Henry, G. W. 1999, PASP, 111, 845

\section{REFERENCES}

Holman, M. J., \& Murray, N. W. 2005, Science, 307, 1288

Holman, M. J., et al. 2006, ApJ, 652, 1715

2007, ApJ, 664, 1185

Ikoma, M., Guillot, T., Genda, H., Tanigawa, T., \& Ida, S. 2006, ApJ, 650, 1150

Kallrath, J., \& Milone, E. F. 1999, Eclipsing Binary Stars: Modeling and Analysis (New York: Springer)

Mandel, K., \& Agol, E. 2002, ApJ, 580, L171

Marcy, G. W., \& Butler, R. P. 2000, PASP, 112, 137

Masana, E., Jordi, C., \& Ribas, I. 2006, A\&A, 450, 735

Mizuno, H. 1980, Prog. Theor. Phys., 64, 544

Perryman, M. A. C., et al. 1997, A\&A, 323, L49

Pollack, J. B., Hubickyj, O., Bodenheimer, P., Lissauer, J. J., Podolak, M., \& Greenzweig, Y. 1996, Icarus, 124, 62

Santos, N. C., Israelian, G., Mayor, M., Rebolo, R., \& Udry, S. 2003, A\&A, 398,363

Sato, B., et al. 2005, ApJ, 633, 465

Seager, S., \& Mallén-Ornelas, G. 2003, ApJ, 585, 1038

Southworth, J., Wheatley, P. J., \& Sams, G. 2007, MNRAS, 379, L11

Sozzetti, A., Torres, G., Charbonneau, D., Latham, D. W., Holman, M. J., Winn, J. N., Laird, J. B., \& O’Donovan, F. T. 2007, ApJ, 664, 1190

Torres, G., Winn, J. N., \& Holman, M. J. 2008, ApJ, in press

Torres, G., et al. 2007, ApJ, 666, L121

Winn, J. N., et al. 2007, AJ, 133, 1828

Yi, S., Demarque, P., Kim, Y.-C., Lee, Y.-W., Ree, C. H., Lejeune, T., \& Barnes, S. 2001, ApJS, 136, 417 